Retrospective Study

\title{
Delayed Initiation of Supplemental Pain Management is Associated with Postherpetic Neuralgia: A Retrospective Study
}

\author{
Xiufang Xing, MD, Kai Sun, MD, and Min Yan, MD
}

From: Department of Anesthesiology and Pain Medicine, Second Affiliated Hospital, Zhejiang University School of Medicine, Hangzhou, China Address Correspondence: Min Yan, MD

Department of Anesthesiology and Pain Medicine

Second Affiliated Hospital Zhejiang University School of Medicine

No. 88 Jiefang Rd.

Hangzhou, China 310009 E-mail: zryanmin@zju.edu.cn

Disclaimer: There was no external funding in

the preparation of this manuscript.

Conflict of interest: Each author certifies that he or she, or a member of his or her immediate family, has no commercial association

(i.e., consultancies,

stock ownership, equity

interest, patent/licensing arrangements, etc.) that might

pose a conflict of interest

in connection with the

submitted manuscript.

Manuscript received: o6-11-2019

Revised manuscript received: 07-15-2019

Accepted for publication: 07-31-2019

Free full manuscript: www.pain physicianjournal. com
Background: Acute pain is a risk factor for developing postherpetic neuralgia (PHN), the most common complication of herpes zoster ( $\mathrm{HZ}$ ). Supplemental analgesics are frequently used in the treatment of acute herpetic pain. However, there are insufficient data regarding when to begin supplemental analgesics, and it is unknown whether the delayed use of supplemental analgesics increases the risk of PHN in high-risk patients.

Objectives: This study aimed to evaluate the association between initial time of supplemental pain management and the risk of PHN in high-risk patients.

Study Design: Retrospective study.

Setting: The Department of Anesthesiology and Pain Medicine, Second Affiliated Hospital, Zhejiang University School of Medicine.

Methods: We performed a retrospective study between May 13, 2017 and August 8, 2018 in our clinic. Multivariable logistic regression analysis was conducted to examine the independent factors associated with PHN. Supplemental pain management was defined as any use of opioids, tricyclic antidepressants, or nerve blocks. A subgroup analysis was conducted in patients who received supplemental pain management within the first 30 days of onset. According to the initial time of supplemental pain management, patients were divided into 2 groups: the early treatment group ( $\leq 14$ days), and the late treatment group (> 14 days). The clinical outcomes in these 2 groups was compared for propensity score (PS) matching.

Results: A total of 134 patients with $\mathrm{HZ}$ aged 50 years or older with moderate to severe pain were enrolled in this study. The delayed initiation of supplemental pain management ( $>14$ days) (odds ratio, $4.11 ; 95 \%$ confidence interval, $1.69-9.92 ; P=0.002$ ) and severity of rash (odds ratio, 2.93; $95 \%$ confidence interval, 1.22-7.01; $P=0.016$ ) were independent factors associated with PHN. In the subgroup analysis, after PS matching, there were no significant differences in the baseline clinical parameters between the early and late treatment groups. The incidence of PHN was significantly lower in the early treatment group than the late treatment group $(36.4 \%$ vs. $72.7 \% ; P=0.015)$. Reduction in pain was also greater in the early treatment group.

Limitations: The findings identified in the present study are specific to the patients who were relatively older and with moderate to severe pain. It is impossible to determine from our study whether younger individuals and individuals with mild acute pain will benefit from early supplemental treatments. Furthermore, because of the retrospective nature of the study, there may be some confounders that could not be controlled. Further prospective studies with larger sample sizes and longer follow-up periods are needed.

Conclusions: The early use of supplemental pain management may decrease the risk of PHN. It might therefore be beneficial to consider administering supplemental pain management earlier in older patients with moderate to severe acute herpetic pain.

Key words: Herpes zoster, postherpetic neuralgia, analgesia, opioid, nerve block, tricyclic antidepressant

Pain Physician 2020: 23:65-72 
H

erpes zoster $(\mathrm{HZ})$ results from the replication of latent varicella-zoster virus in sensory neurons (1). Although rash is its most prominent feature, both acute and chronic zoster-associated pain is its most debilitating symptom $(2,3)$. The persistent pain after the rash onset $>90$ days is known as postherpetic neuralgia (PHN) (4).

Acute pain occurs in at least $95 \%$ of patients over 50 years of age with $\mathrm{HZ}$. At least $40 \%$ of patients with pain consider the pain to be severe (5). It is important to understand that the effect of $\mathrm{HZ}$ on quality of life in older patients is directly related to pain intensity (68). The primary goal of treating $\mathrm{HZ}$ in the elderly is to reduce or eliminate acute pain and prevent $\operatorname{PHN}(6,8)$. Antiviral agents and corticosteroids are the mainstay of therapy to accelerate healing of $\mathrm{HZ}$ and relieve pain, as well as other personalized pain-management modalities as needed $(9,10)$. For mild pain, analgesics such as paracetamol and ibuprofen are appropriate. For moderate to severe pain, if antiviral agents combined with oral analgesics are not sufficient, supplemental pain medication should be considered (10). The most effective means of pain management during acute $\mathrm{HZ}$ is still underinvestigated.

Few randomized placebo-controlled clinical trials have found the efficacy of supplemental pain managements such as anticonvulsants, opioid analgesics, tricyclic antidepressants, or nerve blocks in reducing acute pain and on preventing PHN (11-15), but there are insufficient data regarding when to begin supplemental pain medication. In the treatment of $\mathrm{HZ}$, dermatologists tend to hesitate to provide early active management of pain associated with $\mathrm{HZ}(16)$. In most cases with moderate to severe herpetic acute pain, supplemental analgesics may be considered when the pain control with antiviral medication and oral analgesics is inadequate (7). More and more guidelines recommend that early

Table 1. Inclusion and exclusion criteria.

\begin{tabular}{|l|l|}
\hline Inclusion criteria & Exclusion criteria \\
\hline - Diagnosed with herpes zoster & - First treatment time exceeds \\
- Age $\geq 50$ & 14 days after rash onset \\
- Acute pain intensity $\geq 4$ & - Absence of 3-month follow- \\
[evaluated by the numeric & up data \\
rating scale (NRS): $0-10$ reflects & \\
from no pain to maximum & \\
imaginable pain] & \\
- Disease duration $<90$ days after & \\
the rash onset & \\
\hline
\end{tabular}

Acute pain intensity was defined as the most severe pain from the prodromal phase to the first 2 weeks. aggressive pain management should be considered if the baseline pain severity is moderate to severe or if the patient has other risk factors for PHN $(2,17)$, although the evidence was weak. The goal of this study was to estimate the association between initial time of supplemental pain management and the occurrence of $\mathrm{PHN}$ in patients with high risk of PHN.

\section{Methods}

\section{Setting and Patients}

This retrospective study was approved by the institutional review board (IRB) of Second Affiliated Hospital of Zhejiang University, School of Medicine, a tertiary, academic medical center. Considering the retrospective nature of the study, the informed consents from the patients were waived by the IRB.

We reviewed the medical records of patients who were diagnosed with $\mathrm{HZ}$, and who received medical treatment at the Department of Pain Clinic between May 13, 2017 and August 8, 2018. The criteria for inclusion and exclusion are shown in Table 1.

\section{Data Collection and Outcomes}

We collected demographic data and clinical findings such as age, gender, presence of prodromal pain, duration of prodromal pain, dermatome affected, severity of skin lesion, intensity of acute pain, and underlying systemic diseases from the medical records. Acute pain intensity was defined as the most severe pain from the prodromal phase to the first 2 weeks (18). The category and initial time of each treatment modality was also retrieved from the medical record. Choice of treatment was dependent on the patients' clinical status and their symptoms following routine clinical standards. We defined the treatment modality of supplemental pain management as any use of opioids, tricyclic antidepressants, or nerve blocks. Time to treatment was defined as the duration between the onset of rash and the initial use of each treatment modality.

Pain in the affected dermatome was recorded for 3 months after rash onset using the Numeric Rating Scale (NRS-11) pain score either in person or by telephone. PHN was defined as the presence of pain with NRS-11 $\geq 3$ after 90 days of rash onset. A patient with pain intensity of NRS- $11<3$ or a patient whose treatment was terminated owing to pain disappearance was defined as non-PHN (19). Any complication that occurred within 3 months was recorded. All data were collected by a research nurse not involved in the study. 


\section{Statistical Analyses}

Quantitative variables were expressed as means and standard deviations or as medians and interquartile range, as appropriate. Frequency and proportion were used for qualitative data. Quantitative variables were compared between groups by the test or the MannWhitney $U$ test, and categorical data by the Pearson chi-square test or the Fisher exact test.\{

A multivariable logistic regression analysis was applied to identify the independent factors of PHN. The results are presented as odds ratios (ORs) and 95\% confidence intervals $(\mathrm{Cl})$. The receiver-operating characteristic (ROC) analysis was used to obtain a cutoff point to construct categorical variables from continuous data. To evaluate the impact of early and late use of supplemental pain management on clinical outcomes, subgroup analysis was developed in patients who received supplemental pain management within the first 30 days of onset. The incidence of PHN and intensity of pain at different follow-up times were compared based on propensity score-matched samples. Propensity scores were calculated using a multivariable logistic regression model based on the baseline demographic and clinical characteristics difference between the early and late groups. Matching process was performed in 1:1 ratio by the nearest neighboring method.

A $P<0.05$ was considered statistically significant. All analyses were completed with SPSS 22.0 (IBM Corporation, Armonk, NY).

\section{RESULTS}

\section{Independent Factors Associated with PHN}

A total of 370 patients visited the pain clinic for $\mathrm{HZ}$ during the study period. Among them, 159 patients met the initial inclusion criteria. According to the exclusion criteria, 25 patients were excluded. Of these patients, 12 received their first treatment beyond 14 days of rash onset, and 13 patients did not have sufficient follow-up data. A total of 134 patients were included in our analysis (Fig. 1).

The overall demographic and clinical characteristics are shown in Table 2. Seventy-eight women $(58.2 \%)$ and 56 men $(41.8 \%)$, with a mean age of 64.0 years ( \pm 9.2 years; range, $50-89$ years) were included in the study. The most common region of rash localization was thoracic followed by trigeminal. Patients with PHN reported higher intensity of pain $(P=0.029)$, were

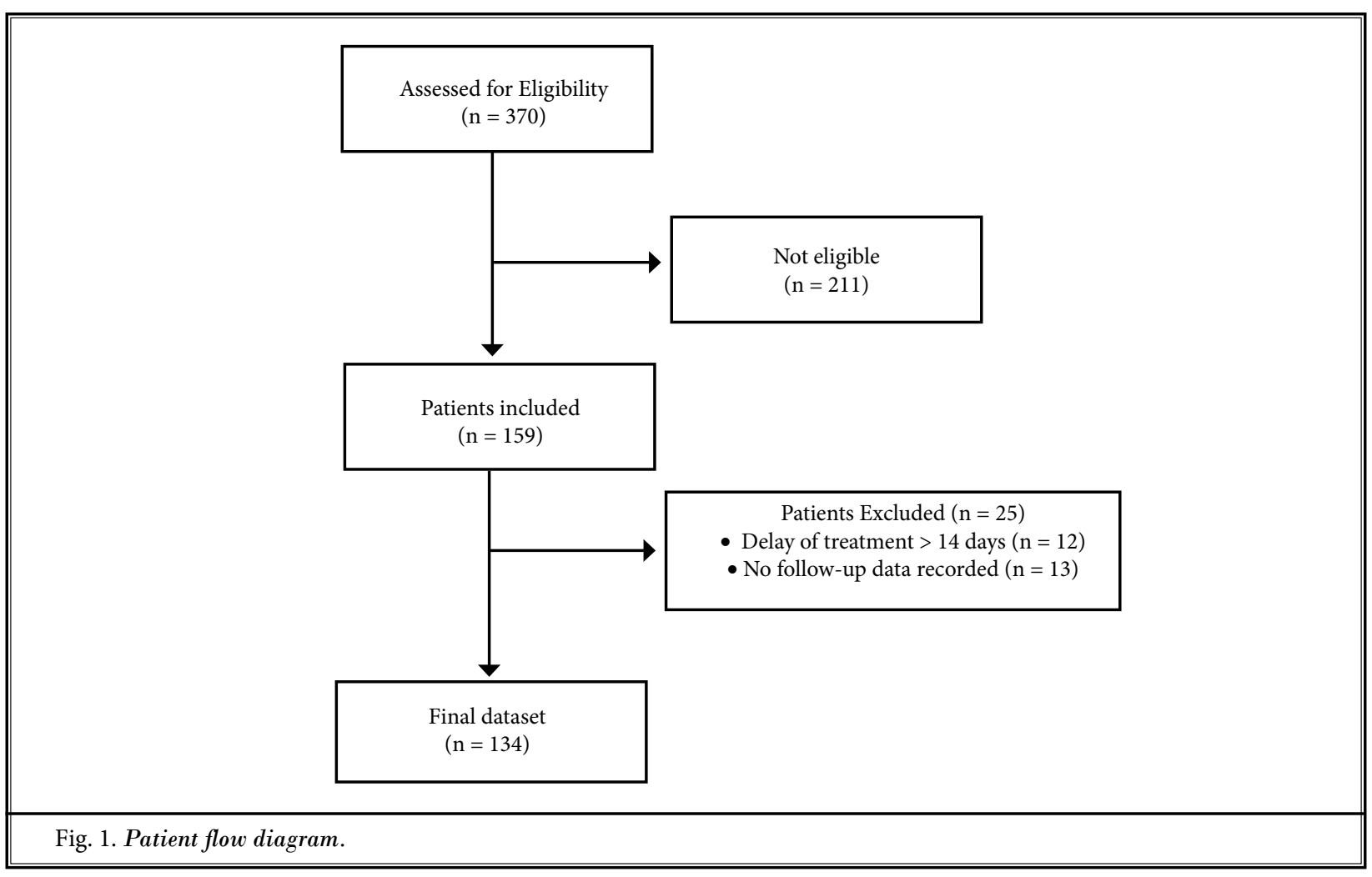


Table 2. Demographic and clinical characteristics of patients with and without PHN.

\begin{tabular}{|l|c|c|c||}
\hline \multicolumn{1}{|c|}{ Characteristics } & $\begin{array}{c}\text { Non-PHN } \\
(\mathbf{n}=\mathbf{7 4})\end{array}$ & $\begin{array}{c}\text { PHN } \\
(\mathbf{n = 6 0 )}\end{array}$ & P value \\
\hline Age, year & $64.0 \pm 9.3$ & $64.1 \pm 9.0$ & 0.932 \\
\hline Male, n (\%) & $31(41.9)$ & $25(41.7)$ & 0.979 \\
\hline Prodromal pain, n (\%) & $54(77.1)$ & $47(81.0)$ & 0.591 \\
\hline Duration of prodromal pain, day & $3(1-6)$ & $3(2-4)$ & 0.390 \\
\hline Intense of prodrome & $4.6 \pm 3.1$ & $4.4 \pm 2.8$ & 0.768 \\
\hline Delay in diagnosis, day & $3(2-5)$ & $3(1-4)$ & 0.046 \\
\hline Rash location, n (\%) & & & 0.667 \\
\hline Trigeminal & $9(12.2)$ & $11(18.3)$ & \\
\hline Cervical & $5(6.8)$ & $6(10.0)$ & \\
\hline Thoracic & $48(64.9)$ & $33(55.0)$ & \\
\hline Lumbar & $9(12.2)$ & $6(10.0)$ & \\
\hline Sacral & $3(4.1)$ & $4(6.7)$ & \\
\hline Severe rash, n (\%) & $26(35.1)$ & $42(70.0)$ & $<0.001$ \\
\hline Acute pain & $7.2 \pm 1.7$ & $7.9 \pm 1.5$ & 0.029 \\
\hline Comorbidities, n (\%) & & & \\
\hline Diabetes mellitus & $5(6.8)$ & $11(18.6)$ & 0.039 \\
\hline Malignant tumor & $7(9.6)$ & $6(10.2)$ & 0.911 \\
\hline Autoimmune disease & $2(2.7)$ & $6(10.2)$ & 0.139 \\
\hline
\end{tabular}

Numerical variables are present as mean \pm standard deviation or medians (interquartile range). Abbreviation: PHN, postherpetic neuralgia.

Table 3. Treatment received during the acute phase of HZ.

\begin{tabular}{|l|c|c|c||}
\hline \multicolumn{1}{|c|}{ Treatment } & $\begin{array}{c}\text { Non-PHN } \\
(\mathbf{n}=\mathbf{7 4})\end{array}$ & $\begin{array}{c}\text { PHN } \\
(\mathbf{n}=\mathbf{6 0})\end{array}$ & P value \\
\hline \multicolumn{2}{|l|}{ Patients of each treatment modality, n (\%) } \\
\hline Antiviral medication & $70(94.6)$ & $57(95.0)$ & 1.000 \\
\hline NSAIDs / paracetamol & $33(44.6)$ & $17(28.3)$ & 0.072 \\
\hline Corticosteroids & $12(16.2)$ & $14(23.3)$ & 0.381 \\
\hline Anticonvulsants & $72(97.3)$ & $54(90)$ & 0.139 \\
\hline Opioids & $41(55.4)$ & $34(56.7)$ & 1.000 \\
\hline Tricyclic antidepressants & $25(33.8)$ & $28(46.7)$ & 0.156 \\
\hline Nerve blocks & $13(17.6)$ & $16(26.7)$ & 0.214 \\
\hline Time to treatment of each treatment modality, day & \\
\hline Antiviral medication & $3(2-5)$ & $3(1-4)$ & 0.225 \\
\hline NSAIDs / paracetamol & $4(2-7)$ & $9(1-14)$ & 0.252 \\
\hline Corticosteroids & $4(3-7)$ & $3(1-6)$ & 0.297 \\
\hline Anticonvulsants & $4(2-7)$ & $3(1-7)$ & 0.471 \\
\hline Opioids & $7(4-14)$ & $15(7-21)$ & 0.017 \\
\hline Tricyclic antidepressants & $8(3-16)$ & $16(8-21)$ & 0.026 \\
\hline Nerve blocks & $7(5-19)$ & $20(14-26)$ & 0.017 \\
\hline
\end{tabular}

Anticonvulsants include gabapentin and pregabalin. Opioids include tramadol and oxycodone with acetaminophen. Tricyclic antidepressants include amitriptyline and duloxetine. Nerve blocks include peripheral nerve block, sympathetic ganglion block and extradural block.

Abbreviation: PHN, postherpetic neuralgia; NSAIDs, non-steroidal antiinflammatory drugs. more likely to suffer from severe rash $(P<0.001)$, and were more likely to have diabetes mellitus $(P$ $=0.039$ ) than patients without PHN. The delay in diagnosis was shorter in patients with PHN than without PHN $(P=0.046)$. The most common choice of analgesic agent used during the acute phase of $\mathrm{HZ}$ was anticonvulsants (94\%) followed by opioids (56\%) and tricyclic antidepressants (40\%), as shown in Table 3. There were no significant differences in the treatment modalities received within the first 30 days of rash onset between patients with and without PHN. The only difference between the groups was that the timing to initiation of opioids, tricyclic antidepressants, and nerve blocks was significantly earlier in patients without PHN than in patients with PHN (7 vs. 15 days, $P=0.017 ; 8$ vs. 16 days, $P=0.026 ; 7$ vs. 20 days, $P=0.017$, respectively).

Independent factors associated with PHN was identified by multivariable logistic regression analysis. We used supplemental pain management to represent the use of opioids, tricyclic antidepressants, and nerve blocks. If any opioids, tricyclic antidepressants, and nerve blocks were used, supplemental pain management was considered used. As summarized in Table 4, the analysis indicated that severe rash (OR, 2.93; 95\% $\mathrm{Cl}, 1.22-7.01 ; P=0.016)$ and delayed initiation of supplemental pain management ( $>14$ days) (OR, $4.11 ; 95 \% \mathrm{Cl}, 1.69-9.92 ; P=0.002$ ) were independent factors associated with PHN.

\section{Characteristics of the Early and Late Supplemental Pain Management Groups}

A total of 92 patients received supplemental pain management within the first 30 days of rash onset. Based on the multivariate analysis and the ROC analysis, patients were divided into 2 groups according to the initial time of supplemental pain management: the early treatment group $(\leq 14$ days, $n=62$ ), and the late treatment group ( $>14$ days, $n=30$ ). Before propensity score matching, the patients in the late treatment group tended to have higher proportion of severe rash, rash localized to the trigeminal region, and diabetes mellitus (Table 5). Because of the baseline heterogeneity between the 2 groups, we generated further propensity score-matched samples. After propensity score matching, no significant differences existed between these 2 groups in any of 
the clinical parameters, except for the initial time of supplemental pain management (Table 5).

\section{Early Start of Supplemental Pain Management Leads to Greater Pain Relief, and Reduction of PHN Presence}

Compared with the propensity score-matched late treatment group, the early treatment group had lower pain intensity after 2 weeks and 3 weeks of rash onset $(4.5 \pm 2.4$ vs. $6.1 \pm 1.9, P=0.025 ; 3.6 \pm 3.2$ vs. 6.2 $\pm 2.2, P=0.003$, respectively) (Fig. 2). The incidence of PHN was significantly lower in the early treatment group than in the late treatment group (36. $4 \%$ vs. $72.7 \% ; P=0.015$ ).

The most common adverse effects were dizziness followed by somnolence, nausea, constipation, and dry mouth. Severe adverse events were not reported in either groups. Although the frequency of adverse effects within the first 30 days of onset were higher in the early treatment group, the differences were not statistically significant (dizziness: $40.9 \%$ vs. $18.2 \%, P=0.099$; somnolence: $13.6 \%$ vs. $4.5 \%, P=0.607)$.

Table 4. Multivariate analysis of PHN.

\begin{tabular}{|l|l|l|l|}
\hline Factor & OR & 95 \% CI & P value \\
\hline Age (per year) & 0.99 & $0.95-1.04$ & 0.775 \\
\hline Delay in diagnosis (per day) & 0.97 & $0.81-1.16$ & 0.733 \\
\hline Severe rash & 2.93 & $1.22-7.01$ & 0.016 \\
\hline Acute pain & 1.13 & $0.84-1.52$ & 0.420 \\
\hline Diabetes mellitus & 2.81 & $0.65-12.03$ & 0.163 \\
\hline $\begin{array}{l}\text { Delayed initiation of } \\
\text { supplemental pain } \\
\text { management (> 14 days) }\end{array}$ & 4.11 & $1.69-9.92$ & 0.002 \\
\hline
\end{tabular}

Abbreviation: CI: confidence intervals; OR: odds risks; PHN, postherpetic neuralgia.

Table 5. Characteristics of patients use supplemental pain management within 30 days after rash onset.

\begin{tabular}{|c|c|c|c|c|c|c|}
\hline \multirow[b]{2}{*}{ Characteristics } & \multicolumn{3}{|c|}{ Before match } & \multicolumn{3}{|c|}{ After match } \\
\hline & $\begin{array}{l}\text { The early group } \\
\quad(\mathbf{n}=62)\end{array}$ & $\begin{array}{l}\text { The late group } \\
\quad(\mathbf{n}=\mathbf{3 0})\end{array}$ & $P$ value & $\begin{array}{l}\text { The early group } \\
\quad(\mathrm{n}=\mathbf{2 2})\end{array}$ & $\begin{array}{l}\text { The late group } \\
\quad(\mathrm{n}=22)\end{array}$ & $P$ value \\
\hline Age, year & $64.8 \pm 10.2$ & $64.3 \pm 7.8$ & 0.787 & $66.1 \pm 10.0$ & $64.0 \pm 8.3$ & 0.454 \\
\hline Male, n (\%) & $23(37.1)$ & $13(43.3)$ & 0.566 & $7(31.8)$ & $9(40.9)$ & 0.531 \\
\hline Prodromal pain, n (\%) & $45(77.6)$ & $26(89.7)$ & 0.243 & $16(72.7)$ & $19(90.5)$ & 0.240 \\
\hline Duration of prodromal pain, day & $4(2-6)$ & $3(2-4)$ & 0.332 & $3(0-6)$ & $3(2-4)$ & 0.990 \\
\hline Intense of prodrome & $4.6 \pm 3.1$ & $4.9 \pm 2.7$ & 0.631 & $4.4 \pm 3.3$ & $4.8 \pm 2.4$ & 0.686 \\
\hline Delay in diagnosis, day & $3(2-6)$ & $3(1-4)$ & 0.252 & $3(2-4)$ & $3(1-5)$ & 0.923 \\
\hline Rash location, n (\%) & & & 0.109 & & & 0.729 \\
\hline Trigeminal & $6(9.7)$ & $8(26.7)$ & & $1(4.5)$ & $3(13.6)$ & \\
\hline Cervical & $5(8.1)$ & $2(6.7)$ & & $3(13.6)$ & $2(9.1)$ & \\
\hline Thoracic & $41(66.1)$ & $12(40.0)$ & & $14(63.6)$ & $11(50.0)$ & \\
\hline Lumbar & $7(11.3)$ & $5(16.7)$ & & $3(13.6)$ & $4(18.2)$ & \\
\hline Sacral & $3(4.8)$ & $3(10.0)$ & & $1(4.5)$ & $2(9.1)$ & \\
\hline Severe rash, n (\%) & $25(40.3)$ & $21(70.0)$ & 0.008 & $13(59.1)$ & $13(59.1)$ & 1.000 \\
\hline Acute pain & $7.7 \pm 1.5$ & $7.8 \pm 1.7$ & 0.727 & $7.7 \pm 1.3$ & $7.4 \pm 1.6$ & 0.502 \\
\hline \multicolumn{7}{|l|}{ Comorbidities, n (\%) } \\
\hline Diabetes mellitus & $5(8.1)$ & $7(23.3)$ & 0.053 & $5(22.7)$ & $3(13.6)$ & 0.698 \\
\hline Malignant tumor & $8(13.1)$ & $2(6.7)$ & 0.488 & 0 & $2(9.1)$ & 0.488 \\
\hline Autoimmune disease & $3(4.9)$ & $2(6.7)$ & 1.000 & $2(9.1)$ & $1(4.5)$ & 1.000 \\
\hline $\begin{array}{l}\text { Time to supplemental pain } \\
\text { management, day }\end{array}$ & $5(3-7)$ & $20(18-24)$ & $<0.001$ & $4(3-8)$ & $20(18-25)$ & $<0.001$ \\
\hline \multicolumn{7}{|l|}{ Clinical outcome } \\
\hline PHN & $19(30.6)$ & $21(70)$ & $<0.001$ & $8(36.4)$ & $16(72.7)$ & 0.015 \\
\hline
\end{tabular}

Numerical variables are present as mean \pm standard deviation or medians (interquartile range).

Abbreviation: PHN, postherpetic neuralgia. 


\section{Discussion}

To our knowledge, this is the first study designed to evaluate the association between timing of supplemental pain management initiation and the risk of PHN. Multivariable analysis in this study revealed that delayed initiation of treatment with opioids, and/or tricyclic antidepressants, and/or nerve blocks is associated with the development of PHN. In patients with similar clinical status, compared with the late use of supplemental pain management ( $>14$ days of rash onset), the early use decreased the risk of PHN.

Patients with acute herpetic pain require urgent analgesics, not only for the relief of symptoms, but also for the prevention of PHN. The main reason for PHN has been shown to be ongoing painful signal input during the acute inflammatory phase, which causes central nervous system changes that generate chronic pain (20). Recently, attempts to reduce the incidence of PHN have been bolstered by the findings of clinical trials that anticonvulsants, opioid analgesics, tricyclic antidepressants, or nerve blocks are effective treatments for HZ (21-23). However, it remains unknown when supplemental pain managements should be started.

In the present study, we retrospectively analyzed 134 elderly patients with moderate to severe herpetic pain at our clinic. Although there were no significant differences in the treatment modalities between patients with and without PHN, the number of days until the start of opioids, tricyclic antidepressants, and/or nerve blocks after rash onset was significantly earlier in the patients without PHN than in patients with PHN. In addition, the multivariable analysis revealed that the delayed use of supplemental pain management was 1 of 2 independent factors associated with PHN.

This study revealed early initiation of supplemental pain managements decreased the incidence of PHN in patients with moderate to severe acute pain. This association was confirmed by propensity score matching analysis. After propensity score matching, the baseline clinical parameters were similar between the early and late treatment groups, but the incidence of PHN was significantly lower in the early treatment group. We speculate that the reason for lower rates of PHN with earlier use of supplemental pain management is because of the following: good pain control during the acute phase reduces the initiation phase of central hyperexcitability evoked by nociception and lessens the likelihood that some subsequent factor(s) maintain abnormal central processing $(18,20)$. As shown in Fig. 2 , the pain intensity was similar at rash onset between the 2 groups. In the early treatment group, after use of supplemental analgesics, the pain relief was fast, and the mean pain intensity was mild at 21 days after rash onset. The pain in the late treatment group continued to be severe until 28 days after rash onset. Although the average pain intensity of both groups fell to mild after 2 months of rash onset, the ongoing severe pain lead by delayed administration of adjuvants resulted in the increased risk of PHN.

Anticonvulsants were the most commonly prescribed analgesics for the treatment of increasing severity of pain at the acute phase of $\mathrm{HZ}$, given the neuropathic component of herpetic pain (24). The preventative effect of gabapentin on PHN promoted Green and Stratman to recommend dermatologists to begin prescribing gabapentin in addition to antivirals in healthy patients with $\mathrm{HZ}$ who are $>50$ years of age and have pain scores $>4$ out of $10(25,26)$. Anticonvulsants were used widely "off-label" in combination with antiviral agents at the time of zoster diagnosis (27), which was consistent with our situation. However, primary care physicians did not routinely prescribe opioids, tricyclic antidepressants, and nerve blocks, especially at the early stage of $\mathrm{HZ}$. The analgesic effect of gabapentin or pregabalin at a starting dosage may be not enough for patients whose acute pain begins suddenly and severely (14). Early use of additional analgesics such as opioids, tricyclic antidepressants, and nerve blocks may provide appropriate pain relief. Thus we defined any use of opioids, tricyclic antidepressants, and nerve blocks as a single treatment modality named supplemental pain management and evaluated whether the early use of supplemental pain management would decrease the prevalence of PHN.

We defined the early use of supplemental pain management as within 14 days of symptom onset based on the ROC analysis in the present study. This timing is consistent with the recommendation that the first 2 weeks after rash onset is the time that treatments have the greatest benefit (28). For these reasons, it is acceptable that the cutoff date was 14 days in the present study and may be in future clinical treatment studies.

This study has several limitations. First, because of the retrospective nature of the study, there may be some confounders that could not be controlled. Second, these findings are specific to the relatively older and with moderate to severe pain. The associations found in this study may differ in younger individuals and individuals with mild acute pain. It is impossible to determine from our study whether such individu- 


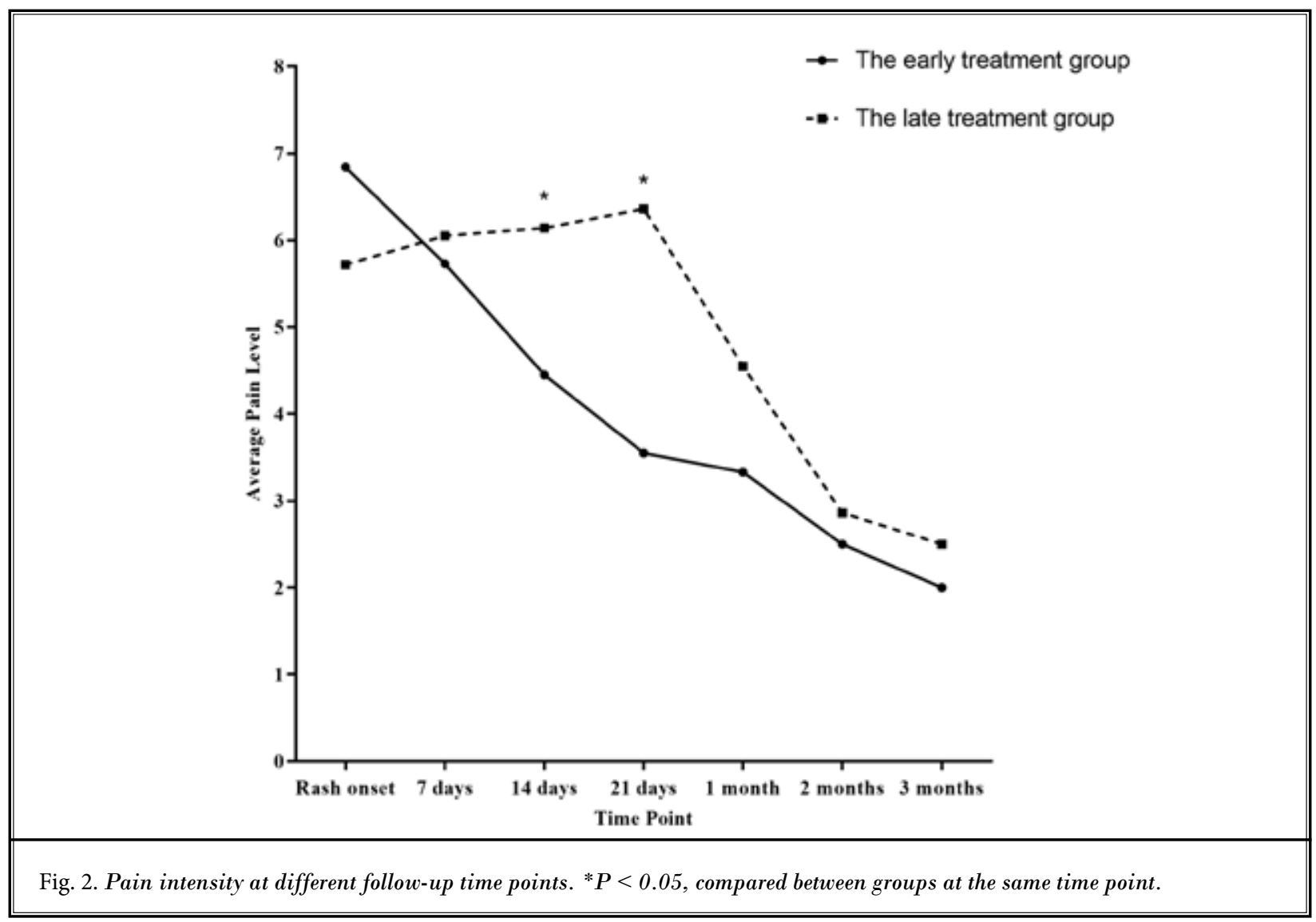

als will benefit from early supplemental treatments or whether antiviral agents with oral analgesics might be efficacious. Third, we defined supplemental pain management as any use of opioids, tricyclic antidepressants, and nerve blocks. The treatments were not uniform. We could not identify the specific effects of each treatment on PHN. Our final limitation is a small sample size and short follow-up period. Further prospective studies with larger sample sizes and longer follow-up periods are needed.

Dermatologists and primary care physicians are the ideal sentinels for the early management of $\mathrm{HZ}$ and the prevention of PHN. They should not only pay attention to expediting rash healing but also to achieving painlessness, considering that pain intensity is the most important determinant of reduced quality of life $(29,30)$. Supplemental pain management such as opioids, tricyclic antidepressants, or nerve blocks were considered to be effective in treating herpetic pain and preventing PHN, but the premise of the validity was early use. Rapid pain control may enhance the probability of a better long-term outcome. Choosing which treatment modality is best should be determined on a case-by-case basis. The treatment of elderly patients is complicated, thus early consultation to a pain therapist may be required (10).

\section{Conclusions}

In summary, for patients aged 50 years or older with moderate to severe pain, delayed initiation of supplemental pain management was associated with PHN. We conclude that supplemental pain management may reduce the risk of PHN when used early in the disease course. 


\section{References}

1. Johnson RW, Rice AS. Clinical practice. Postherpetic neuralgia. N Engl J Med 2014; 371:1526-1533.

2. Cunningham AL, Breuer J, Dwyer DE, et al. The prevention and management of herpes zoster. Med J Aust 2008; 188:171-176.

3. Johnson RW. Consequences and management of pain in herpes zoster.] Infect Dis 2002; 186:S83-90.

4. Johnson RW, Alvarez-Pasquin MJ, Bijl M, et al. Herpes zoster epidemiology, management, and disease and economic burden in Europe: A multidisciplinary perspective. Ther Adv Vaccines 2015; 3:109-120.

5. Gilden DH, Kleinschmidt-DeMasters BK, LaGuardia JJ, Mahalingam R, Cohrs RJ. Neurologic complications of the reactivation of varicella-zoster virus. N Engl J Med 2000; 342:635-645.

6. Schmader K. Herpes zoster. Clin Geriatr Med 2016; 32:539-553.

7. Schmader KE, Dworkin RH. Natural history and treatment of herpes zoster. J Pain 2008; 9:S3-9.

8. Schmader K. Herpes zoster in older adults. Clin Infect Dis 2001; 32:1481-1486.

9. Rajan P, Rivers JK. Varicella zoster virus. Recent advances in management. Can Fam Physician 2001; 47:2299-2304.

10. Werner RN, Nikkels AF, Marinovic B, et al. European consensus-based ( $S_{2} \mathrm{k}$ ) guideline on the management of herpes zoster - guided by the European Dermatology Forum (EDF) in cooperation with the European Academy of Dermatology and Venereology (EADV), Part 2: Treatment. J Eur Acad Dermatol Venereol 2017; 31:20-29.

11. Berry JD, Petersen KL. A single dose of gabapentin reduces acute pain and allodynia in patients with herpes zoster. Neurology 2005; 65:444-447.

12. Ji G, Niu J, Shi Y, Hou L, Lu Y, Xiong L. The effectiveness of repetitive paravertebral injections with local anesthetics and steroids for the prevention of postherpetic neuralgia in patients with acute herpes zoster. Anesth Analg 2009; 109:1651-1655.

13. Makharita MY, Amr YM, El-Bayoumy Y. Effect of early stellate ganglion blockade for facial pain from acute herpes zoster and incidence of postherpetic neuralgia. Pain Physician 2012; 15:467-474.

14. Dworkin RH, Barbano RL, Tyring SK, et al. A randomized, placebo-controlled trial of oxycodone and of gabapentin for acute pain in herpes zoster. Pain 2009; 142:209-217.

15. Bowsher D. The effects of pre-emptive treatment of postherpetic neuralgia with amitriptyline: A randomized, doubleblind, placebo-controlled trial. J Pain Symptom Manage 1997; 13:327-331.

16. Jang YH, Lee JS, Kim SL, et al. Do interventional pain management procedures during the acute phase of herpes zoster prevent postherpetic neuralgia in the elderly? A meta-analysis of randomized controlled trials. Ann Dermatol 2015; 27:771-774.

17. Schmader K. Herpes zoster. Ann Intern Med 2018; 169:897.

18. Haanpaa M, Laippala P, Nurmikko T. Allodynia and pinprick hypesthesia in acute herpes zoster, and the development of postherpetic neuralgia. J Pain Symptom Manage 2000; 20:50-58.

19. Curran D, Schmidt-Ott R, Schutter U, Simon J, Anastassopoulou A, Matthews S. Impact of herpes zoster and postherpetic neuralgia on the quality of life of Germans aged 50 or above. BMC Infect Dis 2018; 18:496.

20. Bennett GJ. Hypotheses on the pathogenesis of herpes zoster-associated pain. Ann Neurol 1994; 35:S38-41.

21. Cui JZ, Zhang XB, Zhu P, et al. Effect of repetitive intracutaneous injections with local anesthetics and steroids for acute thoracic herpes zoster and incidence of postherpetic neuralgia. Pain Med 2017; 18:1566-1572.
22. Lee EG, Lee HJ, Hyun DJ, Min K, Kim $\mathrm{DH}$, Yoon MS. Efficacy of low dose gabapentin in acute herpes zoster for preventing postherpetic neuralgia: A prospective controlled study. Dermatol Ther 2016; 29:184-190.

23. Liang L, Li X, Zhang G, Sun Y, Yu H, Jiao J. Pregabalin in the treatment of herpetic neuralgia: Results of a multicenter Chinese study. Pain Med 2015; 16:160-167.

24. Crosbie B, Lucey S, Tilson L, Domegan L, Kieran J. Acute herpes zoster and post herpetic neuralgia in primary care: A study of diagnosis, treatment and cost. Eur J Clin Microbiol Infect Dis 2018; 37:627-631.

25. Lapolla W, Digiorgio C, Haitz K, et al. Incidence of postherpetic neuralgia after combination treatment with gabapentin and valacyclovir in patients with acute herpes zoster: Open-label study. Arch Dermatol 2011; 147:901-907.

26. Green CB, Stratman EJ. Prevent rather than treat postherpetic neuralgia by prescribing gabapentin earlier in patients with herpes zoster: Comment on "Incidence of postherpetic neuralgia after combination treatment with gabapentin and valacyclovir in patients with acute herpes zoster." Arch Dermatol 2011; 147:908.

27. Jensen-Dahm C, Rowbotham MC, Reda $H$, Petersen KL. Effect of a single dose of pregabalin on herpes zoster pain. Trials 2011; 12:55.

28. Dworkin RH, Johnson RW, Breuer J, et al. Recommendations for the management of herpes zoster. Clin Infect Dis 2007; 44:S1-26.

29. Gross G, Schofer H, Wassilew S, et al. Herpes zoster guideline of the German Dermatology Society (DDG). J Clin Virol 2003; 26:277-289; discussion 291-293.

30. Bader MS. Herpes zoster: Diagnostic, therapeutic, and preventive approaches. Postgrad Med 2013; 125:78-91. 